\title{
Analysis of community chronic disease health management mode under the background of big data
}

\author{
Zehao $\mathrm{Yao}^{1}$, Shihua $\mathrm{Cao}^{1 *}$ \\ ${ }^{1}$ Hangzhou Normal University Qianjiang College
}

\begin{abstract}
In recent years, the "Internet + medical" exploration and the country's vigorously promoted hierarchical diagnosis and treatment system have provided an opportunity to improve the status quo of diabetes. Some scholars have proposed "one-to-one binding community nurses" (Wang Li et al., 2016) and personalized treatment based on big data (He Ting et al., 2016). New chronic disease management concepts such as an integrated chronic disease management model for the elderly based on mobile medical technology (Che Fengyuan et al., 2016). Although different names are used, the core point of view is that patients and community doctors complete the contract, the community doctors will take care of the patients, and the hospital doctors will take care of the patients. The patient's blood glucose data can be shared with relatives and friends, community doctors, and hospital doctors in real time with the help of platform tools such as blood glucose meters, mobile apps, and cloud medical platforms. And community and hospital doctors' feedback on patients can also be sent to patients and relatives and friends in real time, thereby realizing hierarchical diagnosis and treatment of diabetic patients when medical resources are scarce and unevenly distributed. This article refers to this model as the "family-style chronic disease management model". The interaction between patients, relatives and friends, community doctors, and hospital doctors is shown in Figure 1.
\end{abstract}

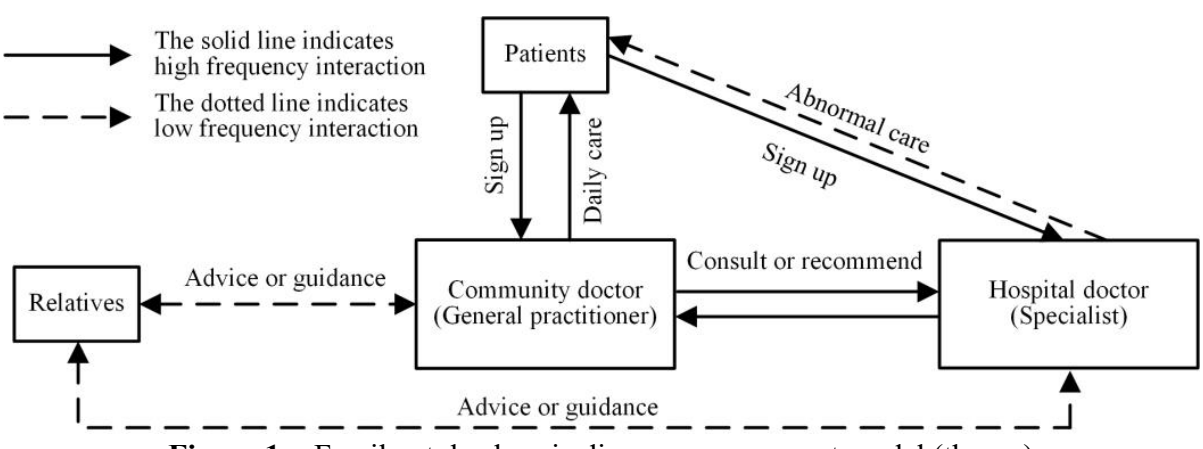

Figure 1. Family-style chronic disease management model (theory)

\begin{abstract}
However, the existing research on the family-based chronic disease management model mostly stays at the level of theoretical explanation, and lacks the practical process and effect research on the family-based chronic disease management model. In order to promote the implementation and optimization of the family-style chronic disease management model, this article attempts to describe the current status and problems of the existing model through the use of tools such as VOSviewer and MATLABR2018b, and provides directions and suggestions for the optimization of the family-style chronic disease management model in my country.
\end{abstract}

\section{Introduction}

Existing research has provided a rich preliminary research foundation for the Internet-based emerging chronic disease management model, but there are still gaps in the following two aspects. First, there is a lack of research on the Chinese context. Due to the huge differences between China's medical system and North America and Europe, the results of empirical research based on North American and European samples may not be fully replicated in the Chinese context. Therefore, the emerging chronic disease management model based on the Internet in the Chinese context involves issues such as which

*yaozehao0807@163.com 
participants, how to divide and interact with each other, and how to evaluate the effectiveness of chronic disease management. Research on chronic disease management based on the Chinese context can help provide operational advice to the Chinese government, chronic disease management companies, doctors, and patients. Second, there is a lack of fine-grained research on the process and mechanism of chronic disease management. Most domestic and foreign studies either outline the main roles and activities of the participants in a rough theoretically,

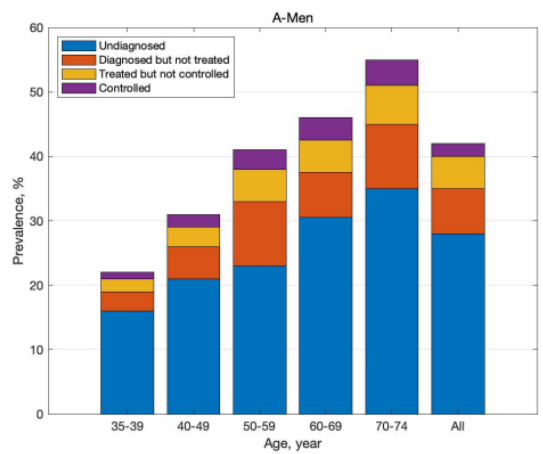

Figure 2. Structural distribution of male diabetic patients

\section{Results}

1. Preliminary preparation: prepare an outline for the interview, determine the selection objects and reference standards of the case. Look up the literature description of the problem at home and abroad.

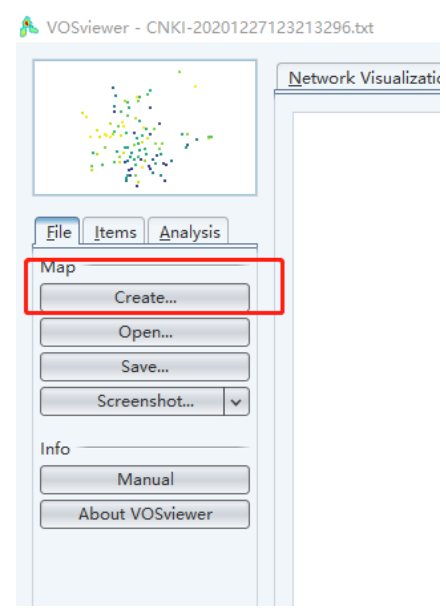

Figure 4. Create a new analysis file or give a statistically significant evaluation of the chronic disease management effects of patients through selfreporting or objective data. There is still a lack of finegrained descriptions of the participation motivation, interaction process and interactive behavior of the participants in the entire chronic disease management model. The implementation process, implementation status, implementation subjective experience and implementation problems of chronic disease management are still black boxes.

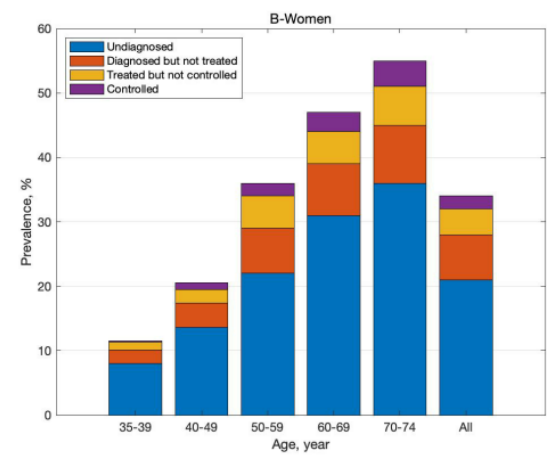

Figure 3. Structural distribution of female diabetic patients

(1) Keyword search for "chronic disease management", download data from CNKI and web ofscience, and export endnote format

(2) In Vscode, maintain and check keywords

(3) Load the export results of HowNet and web of Science in endnotes, and export the RIS output file

(4) Modify the file suffix, change ".txt" to ".ris"

(5) Perform bibliometric operations in the Vosview GUI interface, as shown in the figure below.

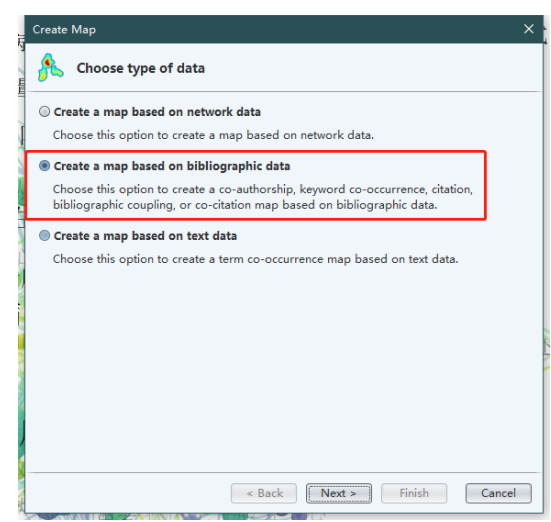

Figure 5. Select input file type 


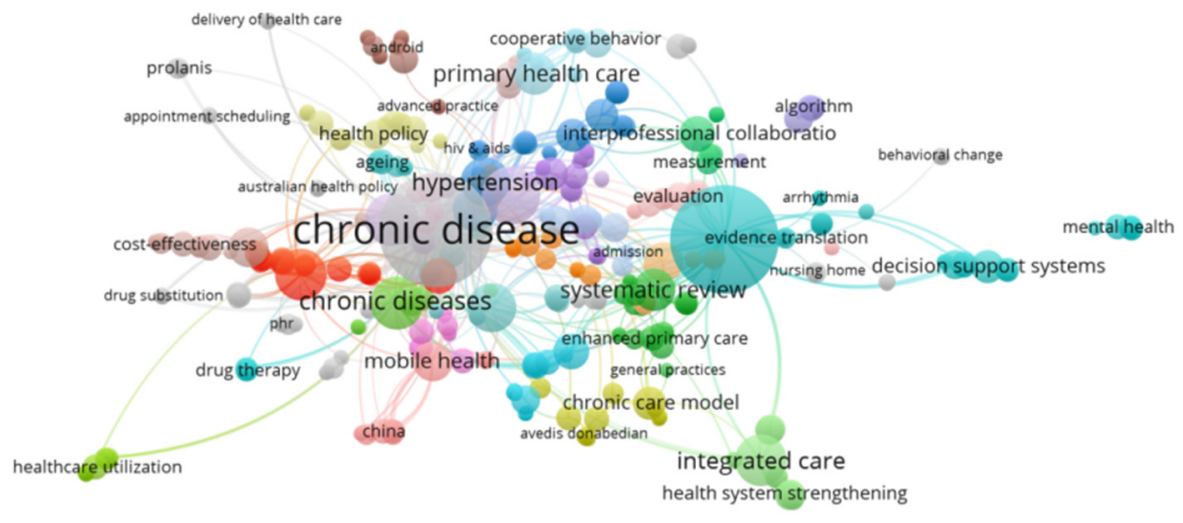

\& vosviewer

Figure 6. Web of science's map of "chronic disease management" keywords

Selection of survey objects: In order to collect true, credible and representative practice data, this article intends to select some third-class hospitals and communities in Hangzhou. The pilot was initiated by the Hangzhou Municipal Government in September 2017 and has achieved good results. In this pilot project, the tool platform provider cooperates with the local government to encourage patients to sign contracts with communities and specialist doctors free of charge, claim smart blood glucose meters and install the WeChat applet "Tangyoukang". After the patient completes the blood glucose measurement, the contracted doctor and the patient's relatives can see the blood glucose data in the small program. If the blood glucose data is abnormal, the contracted doctor will contact the patient one-on-one to understand the cause of the abnormal blood glucose and give a treatment plan. After the free trial of the service for one month, the outpatient clinics of specialist hospitals began to charge 2.5 yuan/day to patients who tried the chronic disease management service. Most of these fees were returned to the specialist in the form of salary. Secondly, the relevant participants in charge of the pilot, including relevant persons in charge of the medical platform, patients, community doctors and specialists, have a strong willingness to participate in the survey, which provides good conditions for the data collection of this study.

2. Research method: This report is designed based on the characteristics of the research question and a single case study. The Internet-based chronic disease management model that this report focuses on is a new phenomenon that emerged under the background of the development of a new generation of health support technology. It still lacks mature theoretical guidance and complete theoretical explanations. A single case study helps this article to go deep into the management practice of representative cases, and combine the rich case background and environmental information to conduct an in-depth analysis and interpretation of the behavior of stakeholders in the chronic disease management model (Eisenhardt \& Graebner, 2007; Walsham, 1995).

Steps: To comprehensively and objectively describe the effects and potential problems of the implementation of the family-style chronic disease management model, collect a wealth of primary and secondary data.

Primary data is mainly obtained through interviews, instant messaging tools, telephone calls, emails, etc. The research team conducted one-to-one interviews with platform tool providers, specialists, community doctors and patients involved in the chronic disease management model in the pilot area.

Second-hand data mainly includes official websites of hardware service providers, news reports on "chronic disease pilot projects" and "chronic disease management" on portal websites such as Tencent, Sohu, and Baidu, relevant industry information reports, and academic peerreviewed journals in both Chinese and English.

\section{Analysis strategy}

The collected data is based on MATLABR2018b, and the number of keywords is output in descending order through the algorithm to output.xls in excel, and then filtered, as shown in Figure 7. 


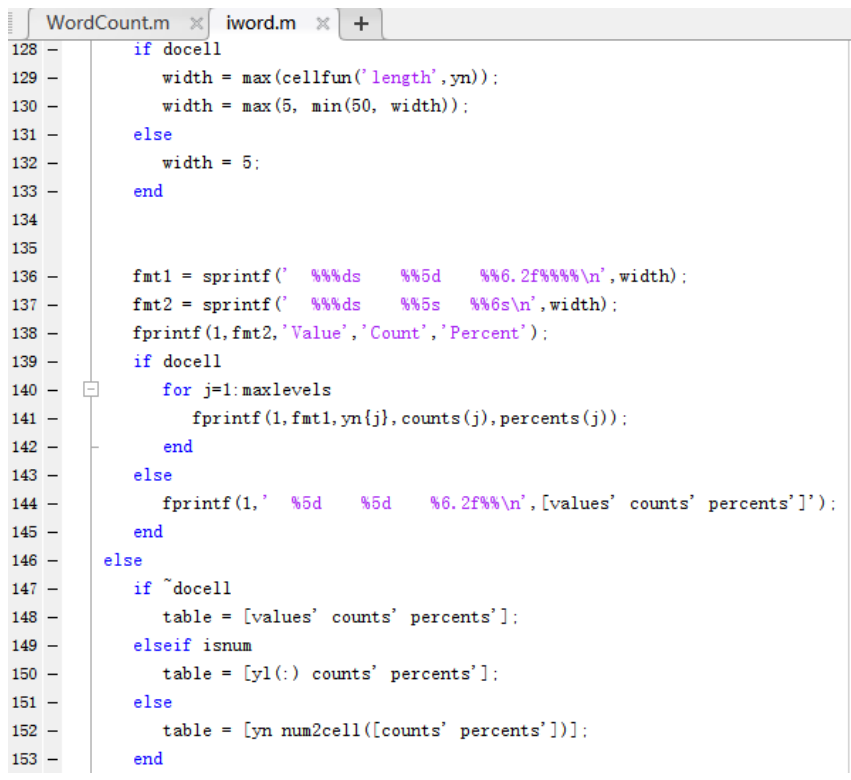

Figure 7. Keywords extraction

Based on the number of occurrences, which is proportional to the size, draw a keyword analysis chart, as shown in Figure 8.

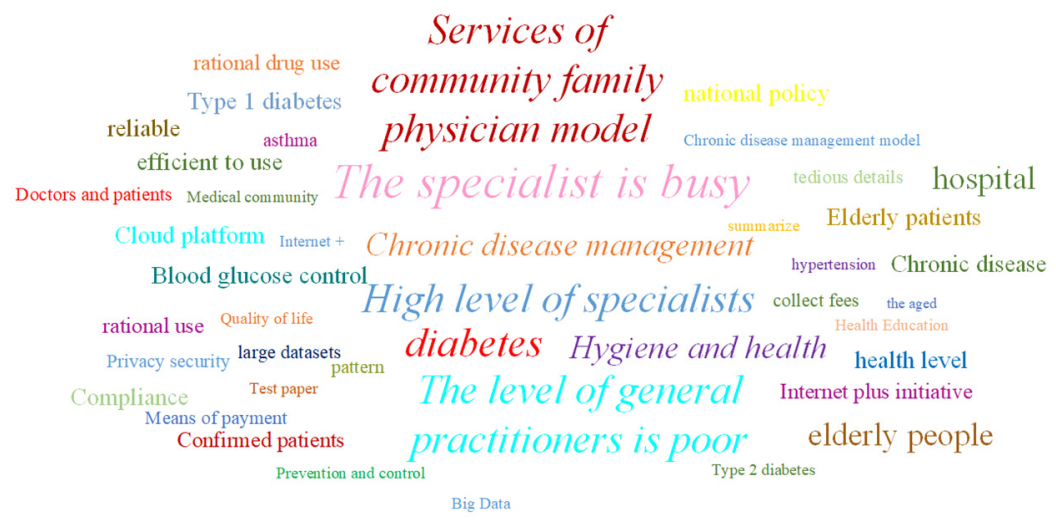

Figure 8. Keyword analysis diagram

\section{Problems in the practice of family blood glucose management}

The practice model of family-style chronic disease management as shown in Figure 9 is drawn. Different from the theoretical model shown in Figure 1, we found that (1) the frequency of interaction between patients and hospital doctors is relatively high; (2) The frequency of interaction between patients and community doctors is relatively low; (3) The frequency of interaction between community doctors and hospital doctors is relatively low. These differences reflect the problems in family-style chronic disease management practices.

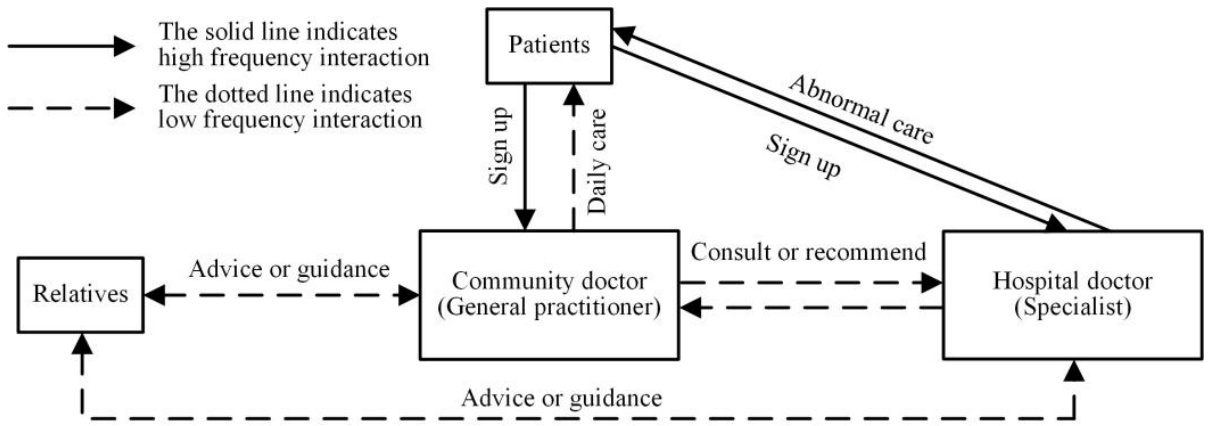

Figure 9. Family-style chronic disease management model (practice) 
1. Weak collaboration between specialists and general practitioners

In order to alleviate the problem of uneven distribution of medical resources, the national policy advocates hierarchical diagnosis and treatment, and encourages the rational division of labor and complementary capabilities between general practitioners and community doctors to jointly achieve daily and abnormal care for patients. Among them, general practitioners are responsible for monitoring and managing the information of chronic disease patients, providing daily health guidance (medication, exercise, diet, etc.) and health knowledge; The specialist doctor is responsible for the management of the patient when the patient's condition changes and the treatment plan or hospitalization needs to be adjusted (Che Fengyuan et al., 2016). At the same time, specialist doctors provide general practitioners with some training and guidance to improve the professional capabilities of general practitioners and enable patients to achieve twoway referral between general practitioners and specialists.

This article summarizes the reasons for the weak collaboration between specialists and general practitioners into the following four aspects.

First, the overall level of general practitioners is poor and they lack understanding of cutting-edge medical knowledge.

Second, patients have not formed the consciousness of "see a general practitioner first if there is a problem" and lack trust in the general practitioner.

Third, the conflict of interest between community hospitals and specialist hospitals hinders the two-way referral of patients. In my country, community hospitals and specialist hospitals maintain independent operations, and there is no standard mechanism that encourages community doctors to recommend critically ill patients to specialists or specialists to take care of stable patients with community doctors. Some doctors rely on personal relationships or sympathy for patients to collaborate, but this collaborative relationship is very fragile and vulnerable to personal work plans.

Fourth, the channels for sharing patient information between specialist doctors and community doctors have not yet been opened up, and it is difficult to conduct indepth collaboration around patients.

2. Patients with poor awareness of paying for chronic disease management

Diabetes is difficult to cure and requires long-term and careful care to slow down the progression of the disease. Most doctors believe that long-term continuous monitoring and management of patients' blood glucose will help keep blood glucose stable, reduce the possibility of serious diseases caused by uncontrolled blood glucose, and in the long run, it can also reduce the cost of medical care for patients.

However, in the current environment, many patients still cannot fully understand the value of chronic disease management services, and their awareness of paying for consultation services provided by doctors is relatively weak. As a result, patients believe that purchasing chronic disease management services has increased the financial burden and tend to stop purchasing services in advance. Due to patients' low awareness of paying for chronic disease management services, it is difficult to maintain long-term payment. On the one hand, it may cause harm to patients' health and bring greater economic burden. On the other hand, doctors feel that their income will decrease and their willingness to participate will also weaken, which will hinder the long-term implementation of family blood glucose management.

\section{Discussion}

Studies have found that the family-based chronic disease management model can indeed effectively control the blood sugar of patients and achieve positive psychological intervention. For doctors, this model can improve the doctor's work efficiency, increase the doctor-patient trust, and increase the doctor's legal income. However, this model also has problems such as weak collaboration between community doctors and hospital doctors and poor patient awareness of seeking medical treatment.

Based on the causes of these problems, this article believes that improving the medical service level and willingness of community doctors is the key to further optimization of the family-style chronic disease management model. Because the improvement of the medical service level of community doctors helps to enhance patients' trust in community doctors, it also helps to improve the collaborative relationship between community doctors and hospital doctors; The gradual change of patients' awareness of seeking medical treatment and payment mainly depends on the popularization and promotion of medical knowledge by community doctors. Therefore, the implementation of the hierarchical diagnosis and treatment policy should pay more attention to the work status and willingness of community doctors. For example, it also provides salary incentives for community doctors to sign contracts and manage patients, strengthen training for community doctors' medical service levels, and provide conditions for community doctors' further training and promotion.

Policy recommendations for general practitioners:

One is to reduce unnecessary social things. Relevant departments must clarify what community doctors should do and which are not undertaken by community doctors. Even if community doctors are required to undertake social work, corresponding remuneration must be paid in time, and they cannot be tied up with the so-called performance pay gimmick. Much of the social work undertaken by community doctors can be separated. Give them to social organizations to complete, so that community doctors can focus on community medical services.

Second, the improvement of community doctors' medical service capabilities should also be emphasized. The improvement of service capacity is a comprehensive project, and the number of health technicians must first be expanded. The original establishment was confirmed in accordance with the planned economic system, and it is no longer suitable for the current primary medical services. The shortage of manpower has now become the norm in various communities, followed by the improvement of comprehensive capabilities, especially the improvement 
of medical services. The current medical consortium construction is a good way.

Although big data analysis can provide services for patients with chronic diseases from various aspects, there are still some problems and shortcomings worthy of attention for our country. On the one hand, since 2009, the US government has established a data open portal site Data.gov, which aims to fully open all data owned by the US federal government and realize data sharing. At the same time, the Australian and American governments are implementing the PCEHR [25] electronic medical file management system, this is a kind of "health management system from birth to grave", which realizes the unification of national electronic medical records, laying a solid foundation for data sharing. However, our country's informatization construction started relatively late, and the understanding of the application of big data in medical treatment is generally insufficient. "Information islands" are widespread, and the corresponding chronic disease data sharing platform is lacking, resulting in a serious shortage of chronic disease data for analysis. On the other hand, with the surging of big data, it also means that data security and privacy security are becoming more and more important. Big data urgently needs to formulate a framework that conforms to its own development and protects users' rights. The Health Insurance Portability and Accountability Act (HIPAA) has passed some privacy security and regulations to ensure that personal information is protected [26]. However, my country has not yet formed a specific legal framework to ensure patient privacy. In addition, our country's technology is relatively backward, and how to effectively use the "big data" method to extract knowledge from massive medicalrelated data and establish an effective, standardized and reasonable new model of chronic disease management still needs in-depth discussion.

\section{References}

1. Che Fengyuan, Ma Yingxia, Li Yuying, Qin Dawei, Kong Guanqing, \& You Shengan et al. (2016). Exploration of an integrated chronic disease management model for the elderly in communities based on mobile medical technology. Chinese Geriatric Healthcare Medicine, 14(4) , 3-6.

2. Dai Yingzi. (2010). the allocation of urban medical resources: imbalance and correction. Urban Development Research, 17(9), 108-112.

3. Gao Yuanyuan, Xie Bo, Sun Zilin, Jia Min, Ye Xiuli, $\& \mathrm{Hu}$ Yuelan et al. (2013). Construction of a new model of hospital-community-patient-volunteer integrated chronic disease management. Chinese General Practice, 16(28), 2624-2626.

4. International Diabetes Federation (DF) Global Diabetes Overview, 8th edition in 2017.

5. He Ting, Liu Xing, Li Ying, \& Yuan Hong. (2016). Research progress in the application of big data analysis in chronic disease management. Chinese Public Health, 32(7), 981-984.
6. Song Yueping, \& Tan Lin. (2006). The accessibility of health and medical resources and the health problems of rural children. Chinese Population Science, 2006(6), 43-48.

7. Tian Hua, Li Shu, \& Zhang Xianglin. (2016). Analysis of the status quo of chronic disease management at home and abroad. China Pharmacy, 27(32), 4465-4468.

8. Wang Li, Chang Lijie, Wu Hao, Liu Lihua, Yu Haiyang, \& Jia Hongyan et al. (2016). Study on the effect of the "one-to-one" binding community nurses participating in the chronic disease health management model of Beijing Fangzhuang Medical Care. Chinese General Practice, 19(30), 3722-3725.

9. Wang Qianjing, Sha Yong, Chen Fang. (2016). Investigation on the health status of rural families in ethnic areas and research on targeted poverty alleviation strategies based on sampling data from Yunnan Province. Guizhou Ethnic Studies, 38(6): 4853.

10. Wang Miao, \& Xing Caixia. (2017). Analysis of urban chronic disease health management in the context of the new medical reform. Medicine and Philosophy, 38(4), 87-90.

11. Yang Yansui, \& Chen Chengcheng. (2017). the restructuring of the medical service system under the silver economy: an analysis of the relationship between long-term care and medical services for the elderly. Journal of the National School of Administration (2), 46-51.

12. Yao Yuan, \& Wang Gaoling. (2017). Reconstruction of the chronic disease management system from the perspective of social management. Chinese Health Service Management, 34(2), 157-160.

13. Yan Jun, \& Chen Yuping. (2010). Do the rural elderly occupy more medical resources? - the policy implications of the age distribution of rural medical expenses. Management World (5), 91-95.

14. Yu Yuxin, \& Yang Dakai. (2008). Theoretical and empirical research on the equity of medical resource allocation in my country. Economic System Reform (6), 160-163.

15. Zhang Yuhui, Wan Quan, Chai Peipei, Guo Feng, Wang Xiufeng. (2017). Research on Diabetes Medical Expenses and Financing Burden in China. Chinese Health Economics. 36(4), 17-19.

16. Zhang Yongjian, Ji Jianlong, Xie Chengyu, Zhou Haiyun, \& Liu Qingyun. (2011). Effectiveness and analysis of chronic disease management in community health service centers. Chinese General Practice, 09(1), 130-132.

17. Zhang Zhongfang. (2017). Research on Medical Security Anti-poverty under the Background of Targeted Poverty Alleviation Policies, Exploration, (2): 81-85.

18. China Industrial Development Research Institute, 2017. 
http://www.chinaidr.com/tradenews/201805/120062.html.

19. Bodenheimer, T., Lorig, K., Holman, H., \& Grumbach, K. (2002). Patient self-management of chronic disease in primary care. JAMA, 288(19), 2469-2475.

20. Braun, Virginia, and Clarke, Victoria. Using thematic analysis in psychology. Qualitatitve Research in Psychology, 2006, 3, 77-101.

21. El-Gayar, O., Timsina, P., Nawar, N., \& Eid, W. (2013). A systematic review of IT for diabetes selfmanagement: are we there yet? International journal of medical informatics, 82(8), 637-652.

22. Goldwater, J. C., Kwon, N. J., Nathanson, A., et al. (2013). Open source electronic health records and chronic disease management. Journal of the American Medical Informatics Association, 21(e1), e50-e54.

23. Johnson, K. B., Patterson, B. L., Ho, Y. X., et al. (2015). The feasibility of text reminders to improve medication adherence in adolescents with asthma. Journal of the American Medical Informatics Association, 23(3), 449-455.

24. Kaplan, S. H., Greenfield, S., \& Ware Jr, J. E. (1989). Assessing the effects of physician-patient interactions on the outcomes of chronic disease. Medical care, S110-S127.

25. Khosravi, P., \& Ghapanchi, A. H. (2016). Investigating the effectiveness of technologies applied to assist seniors: A systematic literature review. International journal of medical informatics, 85(1), 17-26.

26. LeRouge, C., Ma, J., Sneha, S., \& Tolle, K. (2013). User profiles and personas in the design and development of consumer health technologies. International journal of medical informatics, 82(11), e251-e268.

27. Liaw, S. T., Rahimi, A., Ray, P., Taggart, J., et al. (2013). Towards an ontology for data quality in integrated chronic disease management: a realist review of the literature. International journal of medical informatics, 82(1), 10-24.

28. Liu, C. F., \& Kuo, K. M. (2016). Does information overload prevent chronic patients from reading selfmanagement educational materials?. International journal of medical informatics, 89, 1-8.

29. Lorig, K. R., Sobel, D. S., Stewart, A. L., et al. (1999). Evidence suggesting that a chronic disease selfmanagement program can improve health status while reducing hospitalization: a randomized trial. Medical care, 5-14.

30. McDermott, M. S., \& While, A. E. (2013). Maximizing the healthcare environment: a systematic review exploring the potential of computer technology to promote self-management of chronic illness in healthcare settings. Patient education and counseling, 92(1), 13-22.

31. Merolli, M., Gray, K., \& Martin-Sanchez, F. (2013). Developing a framework to generate evidence of health outcomes from social media use in chronic disease management. Medicine 2.0, 2(2).

32. Merolli, M., Gray, K., \& Martin-Sanchez, F. (2013). Health outcomes and related effects of using social media in chronic disease management: a literature review and analysis of affordances. Journal of biomedical informatics, 46(6), 957-969.

33. Merolli, M., Gray, K., \& Martín-Sánchez, F. (2016). Patient Participation in Chronic Pain Management through Social Media: A Clinical Study. In Nursing Informatics (pp. 577-581).

34. Miles, M. B., and Huberman, A. M. Qualitative data analysis: a sourcebook of new methods, 2nd Edition. SAGE Publications, Inc, 1994.

35. Paré, G., Trudel, M. C., Jaana, M., \& Kitsiou, S. (2015). Synthesizing information systems knowledge: A typology of literature reviews. Information \& Management, 52(2), 183-199.

36. Patel, R., Chang, T., Greysen, S. R., \& Chopra, V. (2015). Social media use in chronic disease: a systematic review and novel taxonomy. The American journal of medicine, 128(12), 1335-1350.

37. Rajan, B., Seidmann, A., \& Dorsey, E. R. (2013). The competitive business impact of using telemedicine for the treatment of patients with chronic conditions. Journal of Management Information Systems, 30(2), 127-158.

38. Strauss, A., and Corbin, J. Basics of Qualitative Research: Techniques and Procedures for Developing Grounded Theory. Thousand Oaks, CA: Sage Publications, 1998.

39. Tang, P. C., Overhage, J. M., Chan, A. S., et al. (2012). Online disease management of diabetes: engaging and motivating patients online with enhanced resources-diabetes (EMPOWER-D), a randomized controlled trial. Journal of the American Medical Informatics Association, 20(3), 526-534.

40. Wells, S., Rozenblum, R., Park, A., Dunn, M., \& Bates, D. W. (2014). Organizational strategies for promoting patient and provider uptake of personal health records. Journal of the American Medical Informatics Association, 22(1), 213-222.

41. Yin, R. K. Case Study Research: Design and Methods. Thousand Oaks, CA: Sage Publications, 2003.

Zhou, L., Zhang, D., Yang, C. C., \& Wang, Y. (2018). Harnessing social media for health information management. Electronic Commerce Research and Applications, 27, 139-151. 